\title{
An effective tool to manage the distribution of medicines and monitor the treatment in hospital pharmacies
}

\author{
Gianpaolo Franzoso ${ }^{1}$ \\ 1. Ospedale civile S. Maria Regina degli Angeli. Adria (RO) Italy
}

\begin{abstract}
Introduction: The purpose of the article is to share a modus operandi and a tool that allows the recruitment and management of thousands of patients and their treatment by using a simple software created by the author and made freely available to all colleague-pharmacists. The author, a pharmacist, created this database because there were no tools on the market with all the features needed to manage the treatment of patients and the orders of drugs to ensure continuity of care without waste of public money.

Methods: The data collection is facilitated by the software and allows the monitoring of treatment of the patients and their re-evaluation. This tool can create a table containing all the information needed to predict the demand for drugs, the timing of therapies and of the treatment plans. It is an effective instrument to calculate the optimal purchase of drugs and the delivery of therapies to patients.

Conclusions: A simple tool that allows the management of many patients, reduces research time and facilitates the control of therapies. It allows us to optimize inventory and minimize the stock of drugs. It allows the pharmacist to focus attention on the clinical management of the patient by helping him to follow therapy and respond to his needs.
\end{abstract}

Keywords: Database, drug distribution, drugs delivery, patients management

Abbreviations: Therapeutic Plan (TP); Italian Drug Agency (AIFA); National Health Service (NHS)

Correspondence: gpaolofranzoso@gmail.com

DOI: $10.5210 /$ ojphi.v6i2.5315

Copyright @2014 the author(s)

This is an Open Access article. Authors own copyright of their articles appearing in the Online Journal of Public Health Informatics. Readers may copy articles without permission of the copyright owner(s), as long as the author and OJPHI are acknowledged in the copy and the copy is used for educational, not-for-profit purposes.

\section{Introduction}

In Italy one of the strategies which allows to save a lot of money in the management of therapies is the distribution of drugs through the hospital channel. The definition of the system of reimbursement, distribution, as well as the fixing of the price of the medicine are determined through complex procedures. In the Italian context the Italian Drug Agency (AIFA) is in charge of this decision path. In Italy, drugs prescribed by the National Pharmaceutical Formulary of medicines reimbursed by the National Health Service (NHS) are classified in group A (which becomes group $\mathrm{H}$ when medicines are issued by NHS through the hospital channel and dispensed in hospitals). Alternatively, the medicines can be classified in group C. The medicines reimbursed by the NHS include essential medicines for the treatment of chronic diseases, reimbursed for each therapeutic indication authorized. In some cases there is an AIFA Note, that limits the reimbursement to only some of those indications. Consequently, the class A drugs whose therapeutic indications are not included Online Journal of Public Health Informatics * ISSN 1947-2579 * http://ojphi.org * 6(2):e183, 2014 
in these Notes are charged to the patient. Given the national legislation regulating the reimbursement of medicines and their delivery system, is possible to identify different modes of delivery of medicines in the NHS to patients. Drugs are prescribed in two ways: either by prescriptions by general practitioners or by treatment plans given by medical specialists working in public hospitals. While the first case involves the dispensing of drugs to the patient through contracted pharmacies, in the second case the dispensing of medicine is undertaken directly by health facilities and hospitals through direct distribution. Alternatively, agreements are reached with contracted pharmacies on the distribution account. A recent law has introduced direct distribution as an alternative system for the distribution of medicines, as opposed to the conventional one.

The hospital pharmaceutical management deals with the administration of medicines in hospital pharmacies in the National Health Service. However, for the purposes of monitoring of hospital pharmaceutical expenditure, the consumption of medicines in hospitals also includes prescribed medicines in class $\mathrm{H}$ and class $\mathrm{C}$.

In the case of $\mathrm{H}$ and $\mathrm{A}$ class medicines purchased by public hospital pharmacies, the cost incurred by the NHS coincides with the price resulting from a tender for the purchase or defined as a result of direct negotiation between the hospital pharmacy and the pharmaceutical company.

In the case of medicinal products in class $\mathrm{C}$, the price is defined by the pharmaceutical company and not published but is simply communicated to AIFA.

The governing of national pharmaceutical expenditure essentially makes use of four instruments which are:

1. fixed ceilings of pharmaceutical and hospital expenditure

2. monitoring of pharmaceutical expenditure

3. allocation of a budget to each pharmaceutical company

4. shelf procedures of the pay-back for the benefit of the Regions

One of the tasks undertaken solely by hospital pharmacies is the revision/update of the Regional Therapeutic Handbook and the development of guidelines to address the prescriptive activity [1].

Higher discounts are available to hospitals when ordering drugs from pharmaceutical companies. Distribution directly through the hospital is, therefore, a method of economizing for the National Health Service. The advantages of centralized distribution are also passed on to the patient who is monitored by only one pharmacist throughout the period of therapy. This allows us to establish a relationship of mutual interaction that ensures proper monitoring of therapy. The relationship with the hospital pharmacist allows the patient to have a reference person who can answer questions about treatment. Hospital pharmacist can interface with the doctor who is treating him, resolving any problems that may arise during therapy. The hospital pharmacist is a professional who can interact with doctors gaining valuable information regarding the various therapies and can get in touch with doctors picking up any suggestions that may emerge from the point of view of the prescriber. In the case of innovative therapies the hospital pharmacist can share opinions with the doctor and represent a common link between medical specialist - innovative therapy - the patient. 


\section{Methods}

In the service of the hospital drug distribution in Adria (Rovigo, Italy) medication is managed in the categories previously mentioned.

1. Drugs that must be distributed by hospitals (group $\mathrm{H}$ ).

2. Medications distributed from both hospitals and pharmacies in the territory on restricted medical prescription (group A that are of recent marketing).

For new drugs the Italian Drug Agency establishes special modules to monitor the prescribing indications for use and the duration of therapy called the Therapeutic Plan (TP). It contains patient information, diagnosis, medication, dosage and duration of treatment. The Italian Drug Agency has also created the AIFA Registers: a second instrument used to monitor the appropriateness of prescribing. To categorize the concept we can say that Therapeutic Plans are regulatory instruments with the objective of ensuring the appropriateness of use of medications, in some cases orientating treatment decisions in favour of the most effective and proven molecules and to precisely define the clinical conditions in which the drugs are paid for by the National Health Service. Therapeutic Plans are required for the delivery of drugs:

- for severe specific diseases often with pronounced economic impact

- of recent marketing

- for which it is necessary to carefully monitor the risk/benefit profile.

Therapeutic Plans have a dual purpose: to address and authorize the General Practitioner to prescribe and as an instrument of drug control by the hospitals. For this reason, the Treatment Plan must be completed correctly in all its sections (including patient demographics, the stamp and signature of the specialist who must be clearly identified). It will include the instructions of the AIFA Notes, the information recorded for each medication and treatment protocols identified by the Regions. The AIFA Registers, on the other hand, represent an advanced tool developed from government defined appropriateness for the prescribing of medication. Generally the products are entered in the registers immediately after their marketing authorization, or after approval of an extension of its indications [2]. The Therapeutic Plan can be filled out only by hospital specialists, and there are restrictions as to those who can prescribe and which centres and doctors may prescribe. There is a list of drugs that are marketed with restricted access programmes, and for different drugs, different ways of distribution and monitoring of the therapy can be specified. Restrictions that require the patient to undergo re-evaluation before the continuation of therapy are often added. At the time of the clinical re-evaluation the specialist will assess the effectiveness of treatment, and establish a new Therapeutic Plan a copy of which is then sent to the hospital pharmacy for reference. For therapies that have no particular restrictions on dispensing, current legislation provides for a maximum therapy delivery of sixty days. In this scenario, finding the data to be sent to the Ministry of Health becomes challenging. The foregoing makes it clear that the direction taken is the one that leads to the identification of the exact number of packs delivered to the individual patient in a given period of time. The test of appropriateness is the second prescriptive requirement and the need to be able to identify the prescriber centre, the doctor who prescribed the medication, the dosage, the adjustments necessary, therapy etc presented itself. The problem over the years was as follows: to ensure the continuity of patient's therapy by ensuring the patient always has access to treatment. The hospital had to ensure this without buying large quantities of the 
drug and without creating large inventories, which constitute a value, and may be a waste of money if the drug isn't used before the expiry date. It might seem logical to order the drug only when the patient has a doctor's prescription. In reality, what might apparently seem simple is not the case. This is due to the fact that often, patients are elderly people who do not go to the doctor for repeat prescriptions because they are convinced that the therapy does not require re-evaluation, or people so busy with their work that they do not realize that they have finished the medication until they are down to the last tablet or vial and discontinue therapy as they would have to visit the doctor to request the renewal of the prescription. This therefore, would be a tool which would allow the collection of all the patients' data, facilitate quick access to information about their treatment as soon as the patients come into the pharmacy or need to call the pharmacy. The tool allows the management of these data to resolve the problems of the patient and of the hospital. It was necessary to make sure that the patient was reminded of the monitoring visit before the end of therapy he was undergoing. It was necessary to ensure the availability of the drug and ensure delivery within 24 hours. The author created a database named GPgest after the author's initials. In this database, patients are entered by recording all data, telephone contact and the data of the therapy at the first visit. The database has a list of medications that the pharmacist himself can update or change at will. The software enables the rapid filling of various fields using drop-down menus. These menus can be implemented to maintain the database. This type of software was not available on the database market. It was necessary to have a tool that could calculate with certainty how long the patient would be covered with the packs or units of medication which were being prescribed, so a formula that allows (on the basis of dosing) for the insertion of the unit of drug delivered was included in the software in order to calculate the date on which the patient will exhaust the supply of medication provided. This is shown in Figure 1.

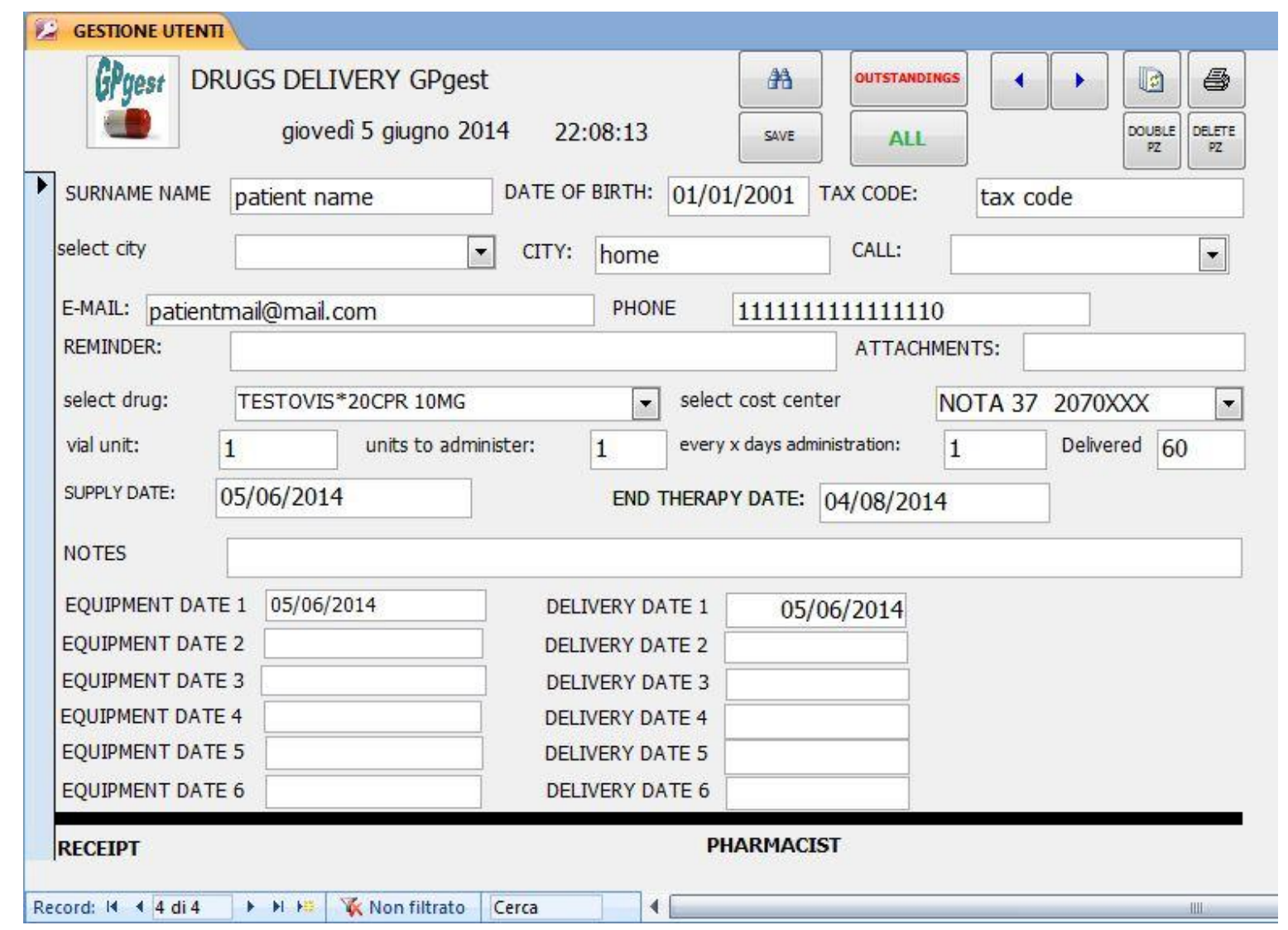


Figure 1: screenshot of database

In this way, when the patient receives the last disbursement, the database will show that for the next request the Therapeutic Plan will expire and advises the pharmacist of the need for re-evaluation.

The patient is entered as follows.

$\mathrm{He}$ is registered in the database. Personal details of the patient are taken.

The TP is checked. At this point checks are made to ensure that the drugs have been prescribed by an authorized practitioner and through an authorized centre. This data is then stored in the database.

Checks are made to ensure that the drug is being used for the correct application and any patient requests are noted, for example, as to the best time to be contacted by phone.

The amount of drug to be delivered is calculated on the basis of the dosage indicated on the Therapeutic Plan.

It will suffice to enter in the "supply date" box the date of dispensing of the drug or the date on which the patient finishes any left-over drug which they might have. Subsequently, the unit of the drug to be dispensed should be inserted. Finally, the frequency with which the patient should take the prescribed dose should be inserted. In Figure 1, the patient takes one pill per day.

For example

If there were 2 pills per day, a 2 is inserted in the box "units to administer" and in the box "every X days administration" 1

If there were 1 phial per week, a 1 would be inserted in the box "units to administer" and a 7 in the box "every $\mathrm{X}$ days administration"

If there were 1 pill every second day, a 1 woud be inserted in the box "units to administer and $\mathrm{a} 2$ in the box " every $\mathrm{X}$ days administration.

Finally the unit of the drug dispensed is inserted. If a pack contains 60 pills and 2 packs are dispensed, suffice to enter 120 in the "quantity" box and the database will enter the date on which the patient will exhaust the drug "end therapy date".

When the sheet is printed, the system prints a sheet which is sent to the patient.

This sheet reminds him when he will run out of the drug that he is receiving and he is informed of the day on which to withdraw the next lot of medication. Figure 2.

The patient must hand in this sheet the next time they collect the drug form the pharmacy.

When the patient returns or requires the sending of the medication to his area of residence, it will be necessary simply to update the disbursement date and amount of the drug sent.

The second time the patient collects the prescription, they will be asked how the therapy is progressing or if they have anything to report. The patient will be asked if they have seen the doctor again and if the dosage has remained the same. If the situation remains unchanged, the stock date and the quantity of the drug dispensed are updated.

On this sheet it is also possible to enter special advice regarding the storage instructions for the drug such as the temperature at which it should be kept. 


\section{HOSPITAL PHARMACY S. MARIA REGINA DEGLI ANGELI ADRIA \\ WE ARE OPEN FROM MONDAY TO FRIDAY 08.00 - 16.00 \\ TELEPHONE 0426940464 \\ FAX $\quad 0426940796$ \\ EMAIL_distribuzione.farmaci@ulss19adria.veneto.it \\ SIR patient name}

We remind you that your supply will exhaust on:

\section{$27 / 07 / 2014$}

We remind you that your therapeutic plan will expire on:

$$
22 / 08 / 2014
$$

continue the therapy as prescribed by physician

The day to withdraw your next disbursement is

$$
20 / 07 / 2014
$$

Figure 2: sheet for patient

\section{HOSPITAL PHARMACY S. MARIA REGINA DEGLI ANGELI ADRIA WE ARE OPEN FROM MONDAY TO FRIDAY 08.00 - 16.00 \\ TELEPHONE 0426940464 \\ FAX $\quad 0426940796$ \\ EMAII distribuzione.farmaci@ulss19adria.veneto.it \\ SIR patient name}

We remind you that your supply will exhaust on:

$$
27 / 07 / 2014
$$

We remind you that your therapeutic plan will expire on:

$$
22 / 08 / 2014
$$

remind to store in refrigerator

The day to withdraw your next disbursement is

$$
20 / 07 / 2014
$$


Figure 3: Insert advice for the patient

The database has designated spaces for the recording of the dates on which the packs of the drug should be prepared and when the patient will collect them.

All this is recorded in a database table that will become the record of disbursements made to each individual patient and become a tool for the pharmacist to manage patient therapy and order him his drug.

The pharmacist will be able to control at fixed time intervals which and how many patients will have exhausted their supply in a given period.

Dates on which the prescription was dispensed can be saved. This allows us to have a clear picture of the patient and makes it possible to understand if he is following the treatment and how he is following it.

The Therapeutic Plan is scanned and stored in a scanned copy with name and date of completion.

For example: John Doe 27.05.2014.

In this way, with a simple search command, all documents relating to a particular patient can be retrieved. This is shown in Figure 4

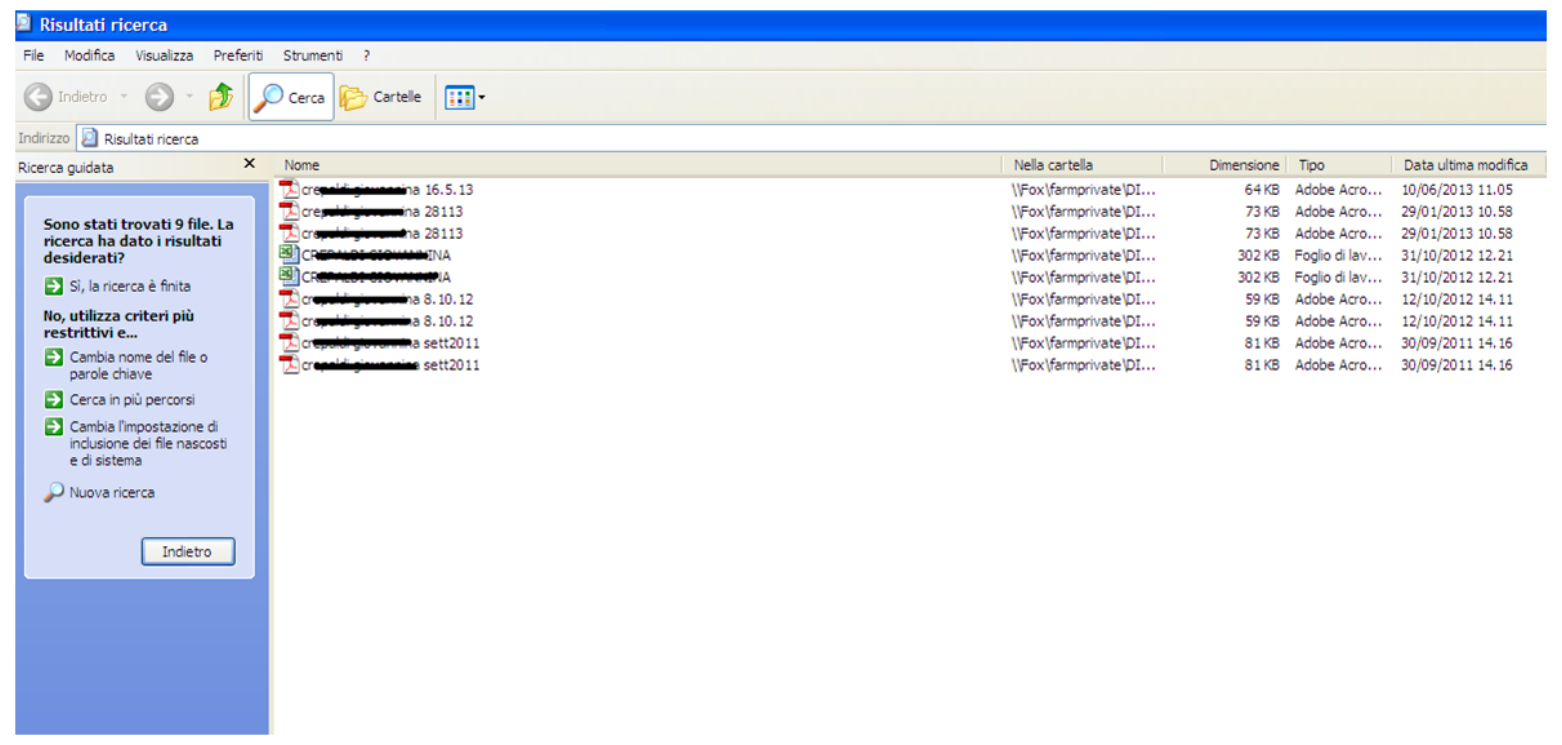

Figure 4: individual patient document

At the end of the working day, all of the prescriptions made on that day are scanned and the bundle of the disbursements saved and renamed in a pre-established manner "dd day-monthyear" to enable to locate the disbursements made on whatever day through a simple search command.

Example:

all the disbursements made on 11/01/2013 are scanned and saved under "dd 11012013";

all the disbursements made on 15/01/2013 are scanned and saved under "dd 15012013" and so on. 
In this way and in a single file it will be possible even months later, to identify how much was disbursed on that particular day. This is shown in Figure 5.

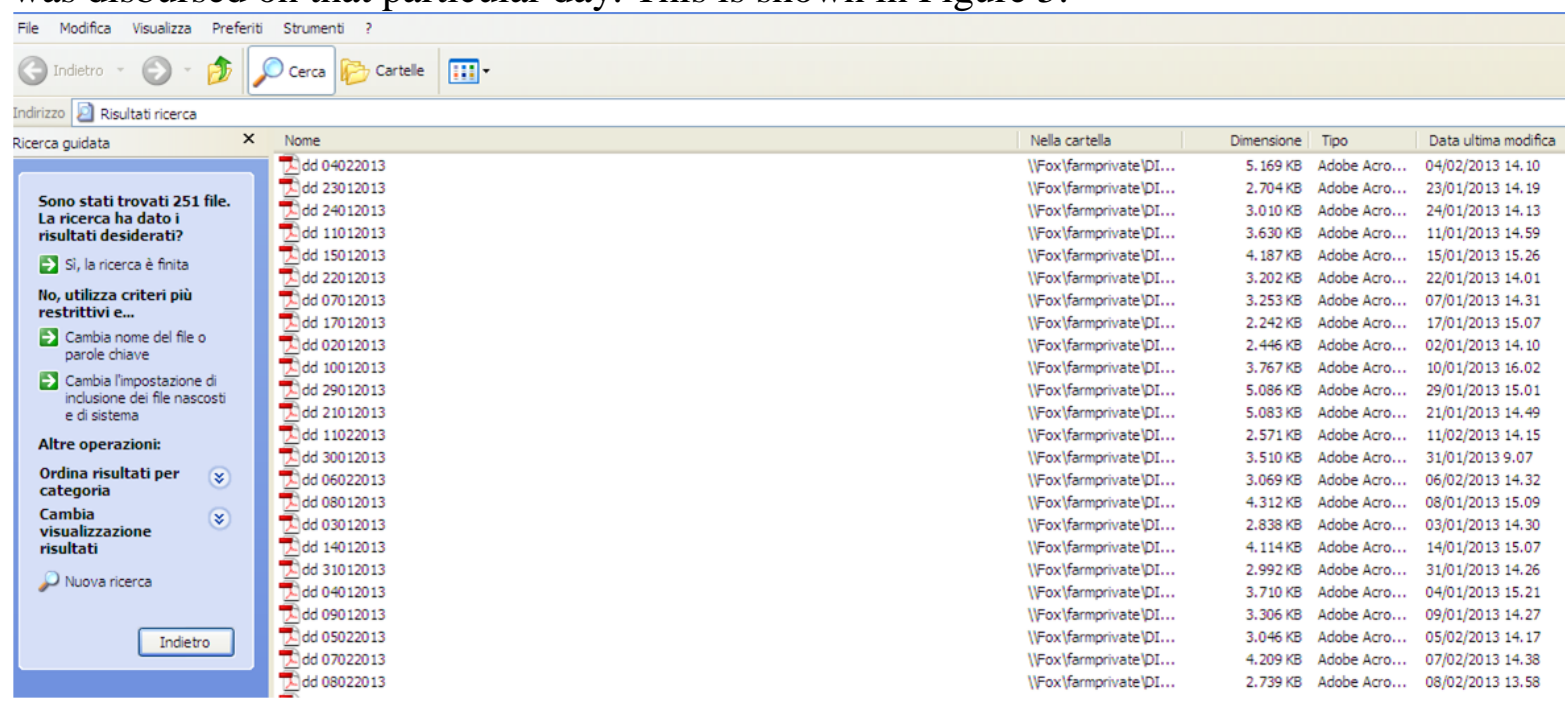

Figure 5: record of disbursements

The database also has a "diary" function which allows the pharmacist to save requests from patients which have to be prepared subsequently. When a patient calls, the pharmacist only needs to look for the name with the designated command and insert the identification number of the pharmacist who takes the call in the field "call". Later on, the pharmacist will prepare this request. With the key "outstandings" the outstanding calls for the pharmacist can be highlighted. This option could be useful for hospitals which send drugs to patients' houses, as happens in the case of this article.

The database is designed to manage all users in the reference area of the hospital in Adria (RO). This territory includes three satellite centres called social health centres. Patients can call the pharmacy and ask for the delivery of the drug to one of these centres which could be closer to their home. Another need therefore, was that for storage and management of all the calls received during the day and to manage all these requests for drugs in the shortest possible time. The database stores all incoming calls during the day and once again at the touch of a button shows which users are in need of medication and had called for medicines (outstanding). A single table incorporates all the data for all of the patients.

As patients are entered into the database a table is created that contains the basic information for each patient, such as medication, the dosage, the centre prescriber, the date on which stocks of medication will run out and the date of expiry of the TP.

With the filters in the programme these tables can be analyzed. By filtering a given drug in a given date range, the drug can be provided at the right time, anticipating the needs of the patient. This table can be used to show how many patients use a drug and predict how many of them will run out of the drug in a given period. In this way, checking in advance, the drug for the individual patient can be ordered. This table can also be exported and, subsequently analyzed with any statistical software to obtain the desired report. With the database, simple tables can be created which contain all the data for each user. Here is how the problem of stock and thus lowered stock value. In three steps data can be extrapolated from the main table which is fed by adding patients into the database. 
First the table can be sorted into columns of drugs.

Filters are used to select the patients being treated with the drug which are then arranged by date of exhaustion of stock.

Thus as shown in Figure 6, it can be estimated how many patients will run out of the drug within a certain time span.

As illustrated in Figure 6 the database shows that there are three patients in the pharmacy with Abilfy $28 \mathrm{cpr} 15 \mathrm{mg}$. Isolating these three patients, it can be noted that of the three, one will finish the drug which he has on the 05/03/2014 but his prescriptions needs renewing.

It could be useful to contact him to see if he has seen his doctor to renew the Therapeutic Plan.

For the other two patients however, a prescription covering at least a month of therapy for both must be foreseen. In this way it is not necessary to buy a random number of packs of the drug, but only the exact quantity necessary in order for the two patients to complete their therapy.

The data was obtained relatively quickly and this allows to order the quantity of medication necessary to cover the two patients highlighted.

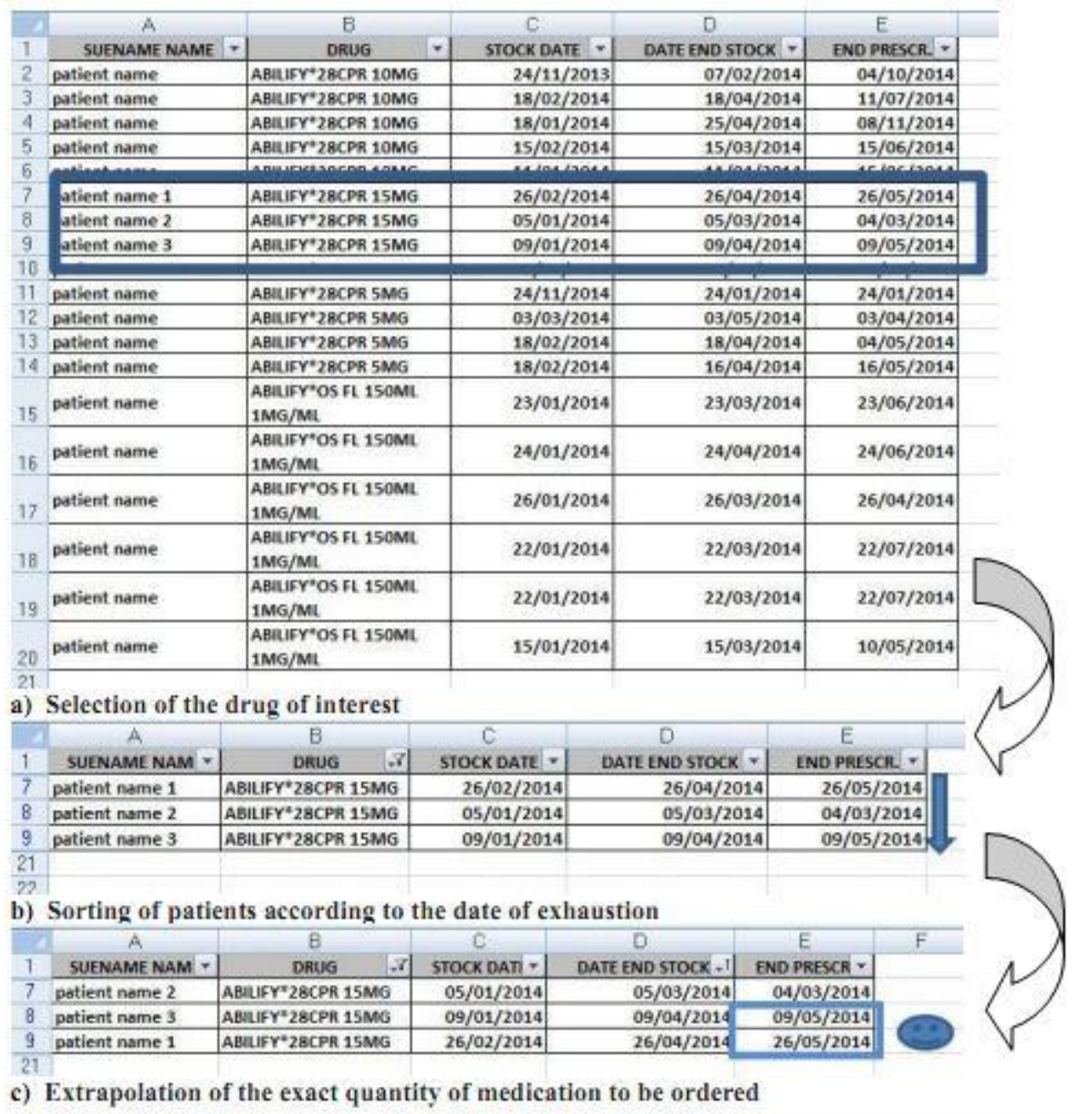

Figure 6: predict the requirements 


\section{Results}

The database allows

- the extraction of a table containing all the information needed to predict how many and which drugs will be needed in a given period of time.

- the programmation of the orders of medications in the appropriate quantity limiting inventory costs and solving the problem of expiry of the drugs.

- the rapid extraction of tables of all users treated with a certain drug or all users who are monitored by a specific prescriber centre. The table thus becomes the repository of all relevant information for studies of drug epidemiology or any other statistical study.

- the creation of a filter table which groups the drugs to be ordered.

- the management of the available budget for the purchase of drugs that is limited and is allocated annually. It was necessary keep within budget and make sure that at the end of the year the stocks were zero. The stocks, in fact, represent a value and the hospital management required that this value be as close to zero as possible.

- not to have large stocks of drugs and not simply to wait for the arrival of the patient but to foresee his needs.

- to predict how much and what medication it will be necessary to buy with targeted orders and without exceeding budget.

- the allocation of the right funds to the right patient in the right moment and in the right amount by ensuring him the therapy without waste.

- the calculation in advance for a given patient of how much medicine he will need without having to buy drugs in excess.

- the avoidance of the purchase of more medicine than is necessary for patients.

- the avoidance of the expiration of the drugs not used.

In this way, in the first three-year period of activity, the ULSS 19 was the only one in the region which achieved an optimization of the costs of direct distribution of drugs, in contrast to a trend on the increase in other institutions as emerges from an official report from the Veneto Region. In Figure 7 a short extracted is cited by way of an example.

In the period studied, all health and hospital facilities (with the exception of ULSS 19 in Adria which shows a decrease of $7.3 \%$ over three years) show an increase in direct distribution expenditure [3].

Obviously these results are the product of team work and guidance on the part of all of the pharmacy staff. The calibration of the quantities of drugs to be ordered has in any event contributed to decreasing costs sustained by direct distribution, and remain within budget without prejudicing the level of patient care. 


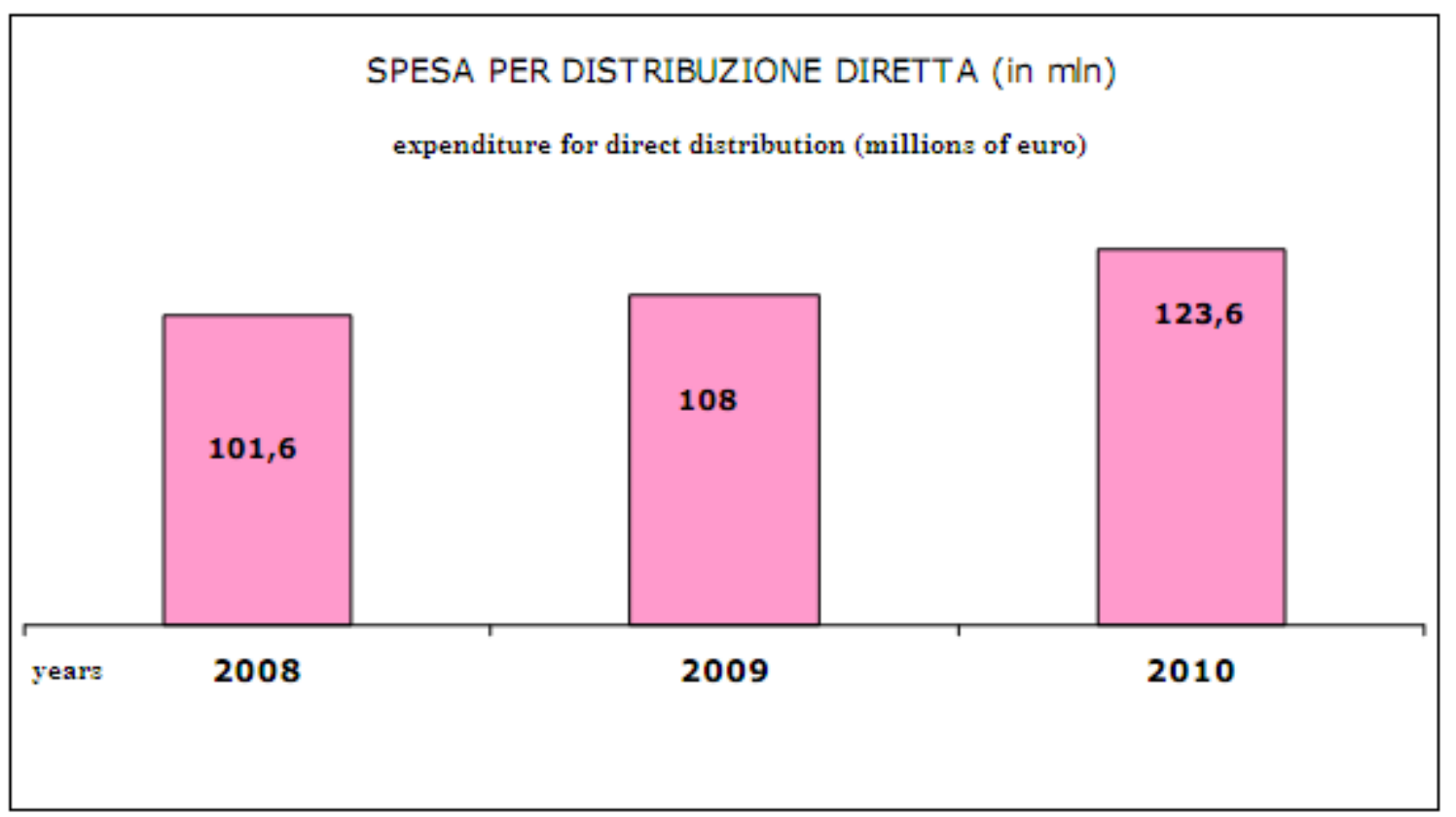

Figure 7: expenditure for direct distribution (Veneto)

\section{Conclusions}

The advantages of this tool for the hospital pharmacy and the hospital have proved to be numerous and can be summarized as follows:

- The hospital can buy only the amount of medication strictly necessary for the management of their patients. This allows a scrupulous and careful use of public funds.

- Stocks of medicines are unnecessary, so the value of stock will be reduced to a minimum, allowing the hospital to move funds from pharmaceutical spending to other areas which may be lacking in resources.

- Vastly reduces the risk of waste of public money by eliminating the possibility that the drugs purchased in excess then expire unused. At the same time, however, therapies will be guaranteed for all patients.

- Improve the management of the patient. The pharmacist can monitor how the patient takes medication and if it is being taken in the right dose. The pharmacist may refer the patient for a check-up with the specialist for adjustment or confirmation of therapy. All this is possible with great immediacy, for example when the patient presents to collect the prescription the pharmacist can immediately check if it is the correct time for withdrawal or if there was some problem with the therapy.

- Anticipating the end of the therapy the pharmacist can also manage access to the pharmacy.

This allows a few pharmacists to manage many patients. 2,000 patients were able to be managed by a single operator. An appointment system was created to allow patients to find the medication ready for collection without long waits. 
As illustrated in Figures $8 \& 9$ the patient is shown what he has to do for the next collection of the drug. If a patient is following a treatment which has just begun or has a long term Therapeutic Plan it will be indicated on the sheet which is given to him to continue with the treatment as illustrated in Figure 8

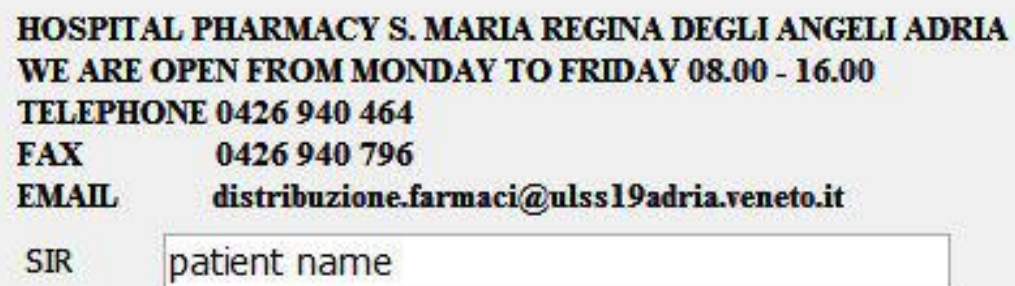

We remind you that your supply will exhaust on:

$$
27 / 07 / 2014
$$

We remind you that your therapeutic plan will expire on:

$$
22 / 08 / 2014
$$

continue the therapy as prescribed by physician

The day to withdraw your next disbursement is

$$
20 / 07 / 2014
$$

Figure 8: patient following treatment

Otherwise, as illustrated in Figure 9 if with the drug prescribed, the patient arrives at the expiry date of the Therapeutic Plan, he is advised at that point that for the next prescription he should contact his Family Practitioner for a check-up.

Availing the patient of this information makes him take responsibility and conscious that the treatment is being monitored in accordance with the Family Practitioner's instructions. The patient who presents to collect his prescription must hand in these forms which were given to him on the preceding occasion. This allows the pharmacist to see if the prescription is being collected at the right time or if it is late rather than early. In tables where it is possible to export from the database all the dates assigned to the patient for successive collections are registered. 


\section{HOSPITAL PHARMACY S. MARIA REGINA DEGLI ANGELI ADRIA \\ WE ARE OPEN FROM MONDAY TO FRIDAY 08.00 - 16.00 \\ TELEPHONE 0426940464 \\ FAX $\quad 0426940796$ \\ EMAIL_distribuzione.farmaci@ulss19adria.veneto.it \\ SIR patient name}

We remind you that your supply will exhaust on:

$30 / 08 / 2014$

We remind you that your therapeutic plan will expire on:

$22 / 08 / 2014$

re-evaluation by physician necessary

$25 / 08 / 2014$

Figure 9: patient requiring medical re-evaluation.

Analysing the tables it is possible to highlight all of the patients who have not presented to collect their prescriptions, to contact them to enquire as to what difficulties they have encountered in following the treatment and the reason for which they have not presented in the pharmacy.

- Often, the Ministry of Health requires the submission by each hospital of the number of patients taking a particular drug, the physician who prescribes them and the centre from which he operates. Switching from paper to computerized management allows the hospital to send data faster if requested. If this were more widely implemented, it could facilitate the monitoring work of the Ministry of Health.

- To date, the Ministry of Health has not focused on the importance of computerized management therapies. For this reason, no software has ever been developed by computer companies.

Software products for hospital facilities are orientated towards the collection of data which are then used for economic and reimbursement purposes between various hospitals countrywide. No software presently monitors the treatment of the patient. The submission of data on the drug consumption of the various patients is limited to a small amount of data that can be sent by each hospital sometimes months in retrospect. Often the member of staff responsible for sending this data is not part of the clinical team but of the administrative one. This prevents the use of that data to check therapies for the benefit of the patient and the preservation of public pharmaceutical expenditure. That is the reason why our corporate

Online Journal of Public Health Informatics * ISSN 1947-2579 * http://ojphi.org * 6(2):e183, 2014 
database and all those currently on the market are not able to predict when a patient will run out of the drug that we are delivering. Knowing in advance for how many patients and for which drugs to use the money available has been the only way that has permitted us to remain within the budget allocated to our hospital by the Ministry of Health.

It was necessary to manage already existing patients, recruit new clients, provide therapy to all patients, lower inventories and communicate patient data quickly subsequent to any inquiry or information requested by the Ministry of Health. This had to be managed by one operator. Unfortunately in Italy the recruitment of staff has been reduced dramatically and the situation as in many Italian hospitals, is very common. With this method, many problems were satisfactorily resolved. The purpose of the article is to share the author's modus operandi in the management of patients in a hospital of small size. Over the past five years more than 1700 patients have been recruited starting from a few hundred existing paperbased medical records. Every year objectives achieved and procedures of computerization have allowed a complete electronic archive of all disbursements to be created that can be called up with a search on the hospital server. A common language and shared resources is an important and necessary step for the development of public health. Shared ideas and speaking a common language can solve many problems which presently mean that public health is singled out as a slow, imprecise, wasteful, bureaucratic slave. Many of these problems are due to the slow introduction of computerization in the public health sector, which coincides with the decline in human resources in hospitals. This example aims to show how, at times, with existing resources many problems can be solved.

The database will be made available free of charge to anyone who wants to use it in their service. It comes with a users' manual in two versions.

Is possible to download the English version and English tutorial and also the Italian version and Italian tutorial on:

https://www.dropbox.com/sh/2r8svetn4srikw1/AABnM4JYNBQWTifwqrka2LT5a?dl=0 or request it by mail from gpaolofranzoso@gmail.com.

\section{Competing interests, disclosure of potential conflict of interest}

Please note that this paper has never been submitted elsewhere in similar form. The sole author is the above-mentioned. I am aware of submission and I agree with it. There are no potential conflicts of interest.

\section{References}

1. National Observatory on the use of medicines. The use of drugs in Italy - Report OSMED 2012. Report for the Italian Drug Agency (AIFA). Rome. Report n. September 2013.

2. National Observatory on the use of medicines. Rome. Report n. September 2013.

3. Italian Republic. The Court of Auditors. Regional section control for the Veneto Region. Deliberazione 247/2012/gest http://www.corteconti.it/export/sites/portalecdc/_documenti/controllo/veneto/2012/delib era_247_2012_gest_e_relazione.pdf (2014 accessed 29 May 2014) 